For these reasons, the at-rest earth pressure was allowed for by assuming that the initial wall normal stress $\sigma_{\mathrm{w}}$ was equal to $K_{0} \gamma d$ and the initial wall shear stress $\tau_{\mathrm{w}}$ was zero, where $K_{0}$ is the coefficient of earth pressure at rest and was taken equal to 0.42 for both dense and loose sand, $\gamma$ is the sand unit weight and $d$ the depth below the surface. These initial stresses were added to the stresses calculated from the load cell readings (whose initial datum was taken with the test tank full of sand) thus giving the total stresses acting on the wall at any stage. Recent measurements of $K_{0}$ for dense sand in the same apparatus, but using load cells designed to measure active pressures, indicate that $K_{0}$ is about 0.2 (Smith, 1972) and that the wall shear stress is not zero but has a value such that the overall angle of wall friction is $-48^{\circ}$. However, these differences between the measured and the assumed $K_{0}$ values would give rise only to small errors in the test data, e.g. for test PE at $\theta=7^{\circ}$, the peak pressure of $52 \mathrm{lb} / \mathrm{sq}$. in. would be changed hy approximately $0.15 \mathrm{lb} / \mathrm{sq}$. in., an error of $0.3 \%$.

The Writer comments on the rapid change in pressure distribution from the assumed initial triangular at-rest distribution $(\theta=0)$ to the parabolic distribution at $\theta=1^{\circ}$ (Fig. 5 (a)). The Authors consider that such a rapid change in pressure distribution is entirely consistent with the pattern of behaviour discussed in the two Papers. Of course, it would be expected that the stress distribution would remain triangular if the mobilized angle of internal friction of the sand were strain independent or if the strains within the mass of sand were uniform. However, it is evident from the strain data presented in Fig. 8 that the strains are highly non-uniform and it is well known that the mobilized value of $\phi$ is highly strain dependent. The strain data at $1^{\circ}$ of wall rotation indicate that there is a shear strain of at least $6 \%$ adjacent to the top of the wall and less than $1 \%$ adjacent to the bottom of the wall. This implies that a significant proportion of the soil shear strength has been mobilized near the top of the wall while only a small proportion of the shear strength has been mobilized near the bottom of the wall. The pressure distribution is therefore parabolic at $\theta=1^{\circ}$.

Finally, the Authors feel bound to emphasize that the classical approach in which a constant angle of internal friction is assumed is very much a mathematical idealization of the complex real situation in which $\phi$ varies with strain. If it is now desired to model reality more closely, equations of strain compatibility and stress equilibrium must be incorporated as well as using a more satisfactory stress-strain law for the soil.

\title{
REFERENCE
}

Smith, I. A. A. (1972). Stress and strain in a sand mass adjacent to a model wall. Forthcoming PhD thesis, Cambridge University.

\section{A microstructural view of the mechanical properties of saturated clay}

CALLADINE, C. R. (1971). Géotechnique 21, No. 4, 391-415.

\section{M. Wood, Cambridge University Engineering Department}

The Author has presented a new 'microstructural' model for the stress-strain behaviour of saturated clay. The analysis in that Paper was, for simplicity, restricted to the axisymmetric conditions of the standard triaxial test.

The Writer has extended the analysis to the more general case of three independent stresses, while still maintaining fixed axes of principal stress. For convenience the experiments with this cxtended model have been limited to 'wet' clays, so that the stress conditions have not reached the cylindrical part of the yield surface, see Fig. 1. 


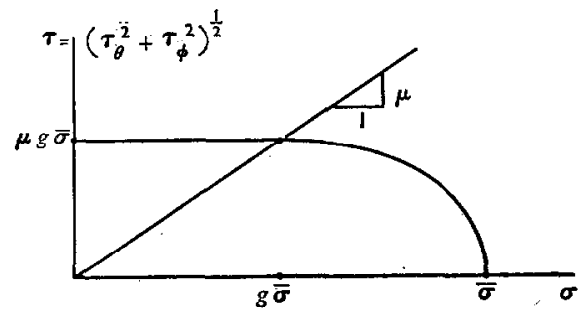

Fig. 1 (above). Section through yield surface for one plane in $\tau$, $\sigma$ space

Fig. 2 (right). Specification of co-ordinate and stress orientation

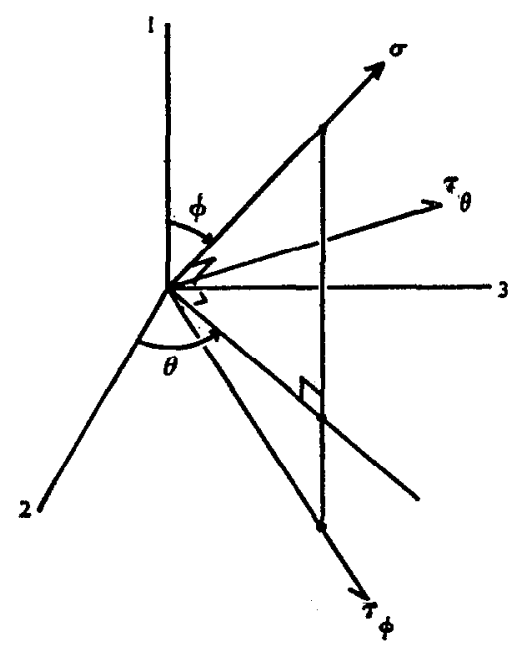

This yield surface for each plane is assumed to be symmetric about the $\sigma$ axis in $\left(\sigma, \tau_{\theta}, \tau_{\phi}\right)$ space (where $\sigma$ is the normal stress, and $\tau_{\theta}, \tau_{\phi}$ are the two perpendicular components of shear stress on any one of the planes of the model, see Fig. 2), and thus has the form of a cylinder with an ellipsoidal cap.

Since, for any given plane, $\sigma, \tau_{\theta}$ and $\tau_{\phi}$ can each be expressed in terms of the principal stresses and the angular location of that plane (Fig. 2), the yield surfaces for each plane in $\left(\sigma_{1}, \sigma_{2}, \sigma_{3}\right)$ space can be established. The ellipsoidal part of the $\left(\sigma, \tau_{\theta}, \tau_{\phi}\right)$ yield surface transforms to an ellipsoid in $\left(\sigma_{1}, \sigma_{2}, \sigma_{3}\right)$ space passing through the point $p=\bar{\sigma}$ on the space diagonal. For an isotropically consolidated material, the ellipsoid for each plane passes through this point. (This is analogous to Fig. 7 (d) in the Author's Paper.)

It can be shown that the principal directions for plastic strains calculated using the principle of normality for the $\left(\sigma, \tau_{\theta}, \tau_{\phi}\right)$ yield loci coincide with the axes of principal stress, and hence that strains in the principal directions can be obtained direct from the $\left(\sigma_{1}, \sigma_{2}, \sigma_{3}\right)$ yield loci. The principal directions for elastic strains also coincide with the principal stress axes. It can further be shown that it is only necessary to consider planes covering one octant of a sphereeach octant will produce the same strains.

It should be emphasized that the principal axes of applied stress and predicted strain will not necessarily be coincident if rotation of the axes of principal stress occurs.

The Writer has performed calculations on an IBM 1130 computer using just ten triangular planes distributed over an octant without bias towards any one of the fixed reference axes $(1,2,3)$. The ten planes had as nearly as possible the same area, but each was assigned a weighting factor equal to the ratio of the arca of the planc to the total arca of all the planes, and this was used when calculating the magnitudes of the strains, which was otherwise done using the same procedure as that of the Author.

A series of computations has been made with different combinations of values of $g$ and $\mu$, see Fig. 1 (where $\mu=\tan \phi$, the coefficient of friction for the material), in an attempt to match data from tests on remoulded kaolin in the Cambridge True Triaxial Apparatus by Pearce (1970). This apparatus permits all three principal stresses to be varied completely independently over large strains while maintaining the uniformity of the sample. 

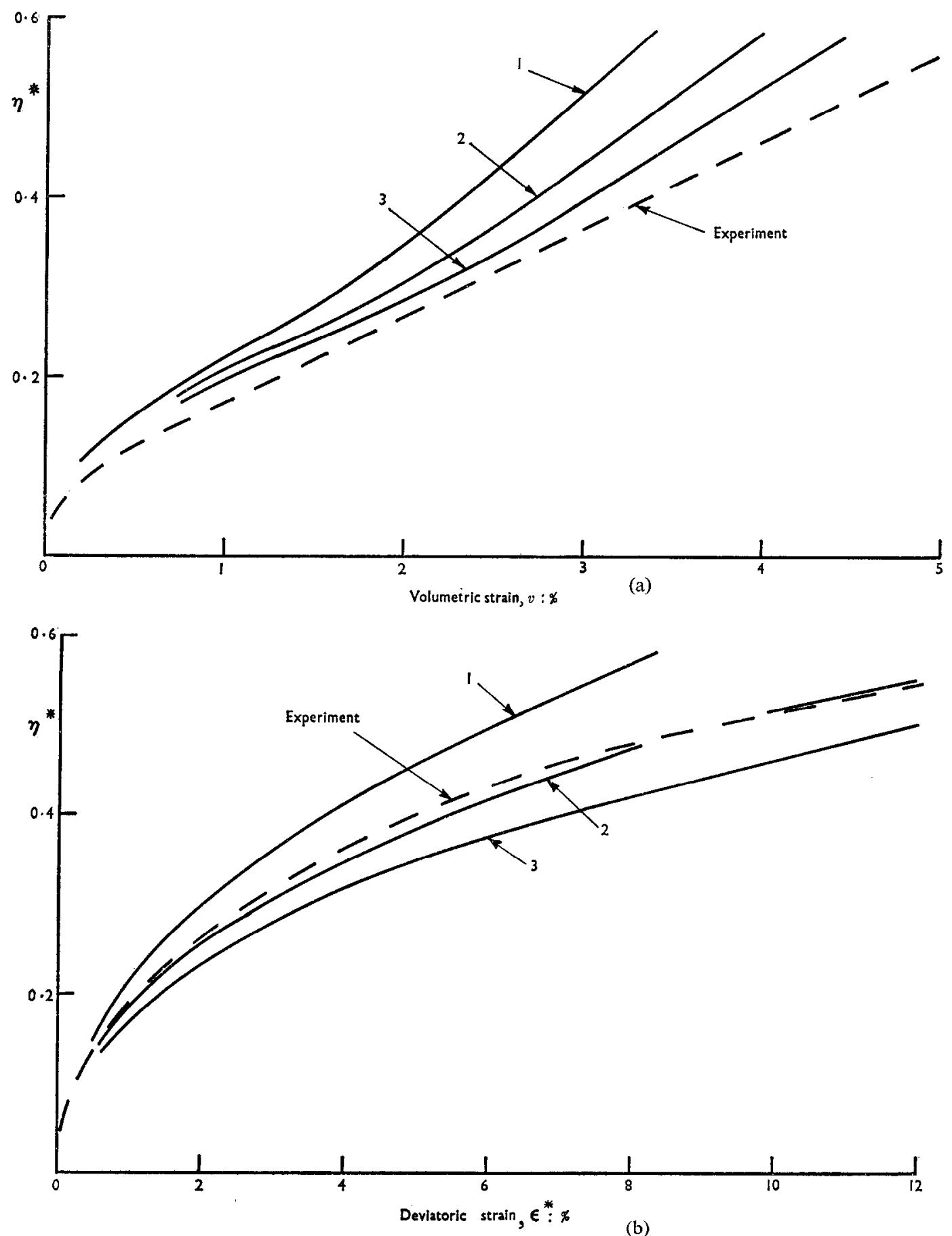

Fig. 3. Comparison of theory with experiment of Pearce (1970) (a) comparison of volumetric strains (b) comparison of deviatoric strains

curve $1: g=0.375, \mu=\tan 26^{\circ}$

(a similar curve is obtained for $g=0 \cdot 40, \mu=\tan 22^{\circ}$ ) 
The effect of varying $g$ and $\mu$ can be seen in Figs 3 (a) and (b) showing the observed and predicted volumetric and deviatoric strains during a test in which the stress ratio $\eta^{*}$ was increased while the mean normal stress $p$ was held approximately constant.

The stresses were increased, from the isotropic conditions at the end of consolidation, in constant ratio such that

$$
\Delta \sigma_{1}^{\prime}: \Delta \sigma_{2}^{\prime}: \Delta \sigma^{\prime}{ }_{3}=1 \cdot 4:-0 \cdot 4:-1 \cdot 0 .
$$

(The Author's reported test comparisons correspond to conditions of conventional triaxial compression or extension in which the applied total stress increments are in the ratio 1:0:0 or $0: 1: 1$ depending on whether the cell pressurc or the axial stress is held constant.)

Note: volumetric strain deviatoric strain $v=\epsilon_{1}+\epsilon_{2}+\epsilon_{3}$ mean normal stress

$$
\epsilon^{*}=\left\{\frac{1}{3}\left[\left(\epsilon_{1}-\epsilon_{2}\right)^{2}+\left(\epsilon_{2}-\epsilon_{3}\right)^{2}+\left(\epsilon_{3}-\epsilon_{1}\right)^{2}\right]\right\}^{1 / 2}
$$

deviatoric stress

$p=\frac{1}{3}\left(\sigma_{1}^{\prime}+\sigma_{2}^{\prime}+\sigma_{3}^{\prime}\right)$

stress ratio

$$
r=\left\{\frac{1}{3}\left[\left(\sigma_{1}^{\prime}-\sigma_{2}^{\prime}\right)^{2}+\left(\sigma_{2}^{\prime}-\sigma_{3}^{\prime}\right)^{2}+\left(\sigma_{3}^{\prime}-\sigma_{1}^{\prime}\right)^{2}\right]\right\}^{1 / 2}
$$

$\eta^{*}=r / p$.

It is apparent that reduction of $g$ and $\mu$ increases all strains, although it was not found easy to obtain close agreement for both volumetric and deviatoric strains using the same combination of values for the parameters.

Increasing the value of $\lambda$ or decreasing the value of $\kappa$ (the slopes of the consolidation and swelling lines in specific volume-log $p$ space) also increases the strains slightly, but all the trials shown here were for $\lambda=0.30$ and $\kappa=0.05$ as obtained by Pearce (1970).

The volumetric strains are rather more difficult to fit than the shear strains; this is in agreement with the Author's Fig. 13 comparing the predictions of the model with the results of tests in the conventional triaxial apparatus. Values of $g=0.375$ or 0.35 give fairly good agreement for $\mu=\tan 23.5^{\circ}$ (the value obtained by Pearce). Similar agreement is found for other True Triaxial Apparatus test results having different values of the intermediate stress ratio.

This model for the stress-strain behaviour of clay is of value in that, unlike most other models based simply on an isotropic plastic material, anisotropic stress history is automatically taken into account. It may be that the expression for determining the magnitude of the strain increments for a given stress increment should be modified in order to obtain improved strain predictions.

The model has the disadvantage of being difficult to apply to predictions of strain controlled tests on soil specimens. Given a set of stresses it is straightforward (as has been shown here) to predict a set of strains. The reverse operation is not so easy and an iterative procedure is required. This may make difficult the application of the model to, for example, finite element analyses of boundary value problems in soil mechanics.

\section{REFERENCE}

Pearce, J. A. (1970). The behaviour of soft clay in a new true triaxial apparatus. PhD thesis, Cambridge University.

\section{R. Calladine}

The Writer has described an extension of the calculations of strains within a sample of soil, which form one aspect of the Paper, to the situation where the values of the three principal stresses are independent. The calculations are naturally much more complex. It is good to know that the results are realistic in the sense that they give fairly good agreement with a test of Pearce (1970) for a simple loading path, provided values of the basic parameters of the model are suitably chosen. 
The Writer's point about the non-coincidence of the principal directions of stress and strainrate calculated from the model if the axes of principal stress rotate relative to the material is important. In most formulations of plasticity theory it is necessary to make assumptions about the coincidence or otherwise of these directions.

It would indeed be possible, as the Writer suggests, to alter the shape of the yield locus in $\sigma, \tau$ space for a slip surface in such a way that the final results fit more closely the experimental observations of Pearce. In the Author's view this sort of modification of the fine details would not be warranted at the present stage. The real strength of the Author's model lies in its ability to make predictions of strain response to arbitrary stress paths. As is clear from the work of Lewin and Burland (1970) among others, more crucial information on material behaviour is obtained by doing experiments in which the stress path in principal stress space is 'angular'. It would be of interest to see how well the Author's model reproduces the strains in tests of this sort when the restriction $\sigma_{2}=\sigma_{3}$ is lifted.

The Writer also takes up the point (p. 405 of the Paper) that in modelling strain-controlled tests it is necessary to use an iterative procedure, and notes that this could lead to difficulties in the implementation of finite-element calculations on boundary-value problems in soil mechanics. Such calculations should never of course be undertaken lightly, wantonly or unadvisedly. Since it is clear that there is still ample room for development of finite-element methods, it might be useful at this stage to outline a new kind of finite-element method which can be devised in the spirit of the Author's model. In this method rigid polyhedral blocks are separated by thin deforming plane layers having appropriate response in terms of $\sigma, \tau$, and so on. Although the angular separation of these deformable slip surfaces within the material would not necessarily be small, the procedure might work well in situations where the 'critical state' was not achieved. A particular advantage of the scheme is that rotation of the axes of principal stress relative to the material would be accommodated naturally.

\section{REFERENCE}

Lewin, P. I. \& Burland, J. B. (1970). Stress-probe experiments on saturated normally consolidated clay. Géotechnique 20, No. 1, 38-56.

\section{Peter Smart, Civil Engineering Department, The University, Glasgow W2}

The Author's Fig. 13 seems to refer to consolidated-drained and consolidated-undrained triaxial tests of Spestone kaolin. The interactions between individual particles of this kaolin have been studied using optical microscopy and scanning and transmission electron microscopy (Smart, 1966, 1967 and 1969). Reconstituted saturated samples were consolidated, uniaxially, to $4 \mathrm{~kg} / \mathrm{cm}^{2}$; some of these samples were then sheared rapidly at an overconsolidation ratio of 2 in a shear box. In addition, examination was made of a failure zone from a triaxial sample from the series tested by Loudon (1967) (also Wroth and Loudon (1967)) and discussed by Roscoe and Burland (1968). The kaolin particles were found to act like hard, rigid, smooth, irregular plates, which readily formed groups of sub-parallel particles called domains, which turned to adopt a preferred orientation under anisotropic stresses, and which, in failure zones, aligned parallel to the zones. Consolidation pressed the domains together to form a turbostratic structure without inter-domain voids. Shearing resulted in the formation of a few voids about $1 \mu \mathrm{m}$ size and in the formation of larger domains (or small shear zones). These observations led to the following hypothesis. "At low over-consolidation ratios, the general stress level during shearing is not much different from that which acted when the domains were formed. These new stresses may be able to reform the domains (so they increase in size and decrease in number) and to hold the domains together and prevent the formation of micro- 


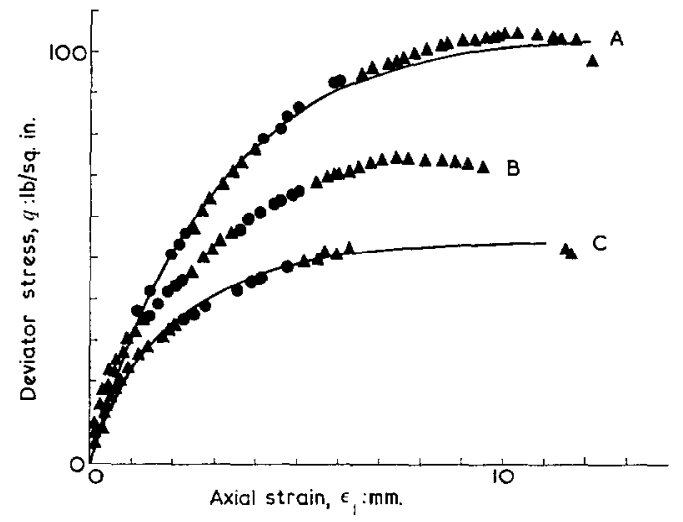

Fig. 1. Plastic portion of some stress-strain diagrams

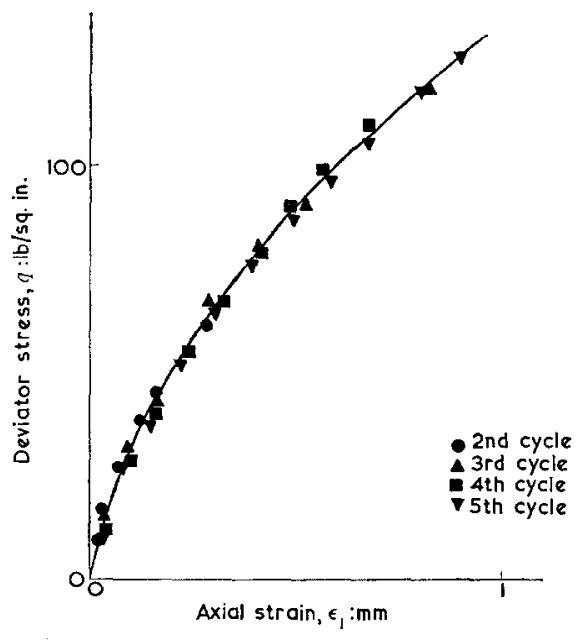

Fig. 2. Elastic portions of some stress-strain curves during reloading

voids. If so, an overall diminution in volume would probably ensue. At high overconsolidation ratios, the pattern of stress will be different; but the stresses on any individual domain may be lower than those under which it was formed. Perhaps, in this case, the domains do not reform but move as rigid blocks. If so, micro-voids would appear between or through the domains, and the overall volume would increase' (Smart, 1969). On this basis, it seems difficult to accept the Author's model for this particular material, unless it can be shown that the material was in a different state in the tests to which he refers.

Since sand particles sometimes break during shear testing, it must be assumed that clay aggregates are also liable to break during shearing. Again, a different analysis is required.

On the other hand, recent work with which the Writer has been associated gives some support to Calladine's model (I. McConnachie, in preparation).

A simple analysis of normally consolidated 'slippery-penny-clays' is possible, as follows. Consider a test in which some of the particles are being turned from their original orientations into some preferred orientation. At any stage of the test, let $f$ be the proportion of these particles which have not yet been turned. Assume that, during an increment of strain $\mathrm{d} \epsilon$, the number of particles turned is proportional to both $f$ and $\mathrm{d} \epsilon$; i.e.

$$
\mathrm{d} f=-a f \mathrm{~d} \epsilon
$$

where $a$ is constant, depending on the material and the type of test. Then

$$
f=A \mathrm{e}^{-a \epsilon}
$$

where $A$ depends on the initial conditions.

Assume that the incremental rigidity of the material is proportional to the number of particles which have not yet turned; i.e.

$$
\mathrm{d} \sigma=C f \mathrm{~d} \epsilon
$$

where $\sigma$ is the stress, and $C$ is a constant. It follows that

$$
\sigma=\sigma_{\infty}\left(1-\mathrm{e}^{-\alpha \epsilon}\right)
$$

$\sigma_{\infty}$ being the ultimate stress. Curve A in Fig. 1 was calculated from equation (4) with an obvious change of notation. The points are observations from a drained triaxial test; the equation seems to approximate the observations fairly well. 
The observations in Fig. 1 are taken from a preliminary series of tests of Devolite, a kaolin from English China Clays, containing $18 \%$ clay and $77 \%$ fine silt. It was supplied in a deflocculated state. Powdered clay was mixed with distilled water; consolidated uniaxially to $30 \mathrm{lb} / \mathrm{sq}$. in.; subdivided; consolidated triaxially to $60 \mathrm{lb} / \mathrm{sq}$. in.; and sheared, drained, at $0.00016 \mathrm{in} . / \mathrm{min}$ nominal strain rate, in triaxial machines at various cell pressures, $60 \mathrm{lb} / \mathrm{sq}$. in. in test A. Small bedding and visco-elastic effects have been ignored throughout the series. Most of the tests were repeated unloading tests, in which the deviator stress was completely removed from time to time. For clarity, the resulting hysteresis loops have been omitted from Fig. 1; but the 'plastic' portions of successive cycles have been distinguished by different symbols, circles and triangles. Test B, Fig. 1 , was at a cell pressure of $40 \mathrm{lb} / \mathrm{sq}$. in.; this test was duplicated by another without repeated unloading, no significant difference being noticed.

For lightly overconsolidated samples, a small correction seems to be required in equation (4)

$$
\sigma=\sigma_{\infty}\left(1-\mathrm{e}^{-a \epsilon}\right)+B\left(\mathrm{e}^{-a \epsilon}-\mathrm{e}^{-b \epsilon}\right)
$$

where $b$ and $B$ are constants. This would result from postulating two turning mechanisms, but see later in this contribution. The form of equation (5) was earlier suggested for top soils by Bekker (1955). Curve C in Fig. 1 was calculated from equation (5). The points are from a test at $30 \mathrm{lb} / \mathrm{sq}$. in. cell pressure and the equation seems to approximate the observations fairly well.

Figure 2 shows the observations of the reloading portions of four consecutive hysteresis loops. For this diagram, the strain has been reckoned zero at the start of each cycle, and the plastic portions of the tests have been omitted. The procedure differed from a repeated loading test in that each cycle was continued to a higher stress than the preceding cycle, and considerable plastic flow occurred during the second portion of each cycle. Thus, the observations have in effect been translated a considerable distance parallel to the (absolute) strain axis. The curve in Fig. 2 was calculated from the equation

$$
\epsilon=D \sigma+E \sigma^{2}
$$

where $D$ and $E$ are constants which depend on cell pressure and possibly other factors. The equation seems to approximate all the sets of observations fairly well.

Equation (6) was originally developed with a view to separating strain into elastic and plastic components, and using only the plastic components in equations such as (4) and (5). However, it may be that at some times the material follows an elastic law such as equation (6) and at other times follows a plastic law such as equations (4) or (5).

The transition between the elastic and plastic portions of the reloading cycles was usually shown by a well-developed knee in the stress-strain diagrams. This is in accordance with the concept of an elastic-plastic material. In this respect, these tests do not contradict Burland's concept of an inncr plastic potential such as $q=$ constant (Roscoe and Burland, 1968). (This series was insufficient to confirm this point.) On the other hand, if there is an outer plastic potential such as $M^{2} p^{2}+q^{2}-M^{2} p_{0} p=0$ (loc. cit.), there should be knees in curves B and C of Fig. 1. Such knees the Writer cannot find; nor is he able to find knees in the corresponding volumetric curves. Furthermore, if curves $\mathrm{B}$ and $\mathrm{C}$ begin as elastic and then become plastic, whereas curve $A$ is plastic throughout, then it may be expected that $B$ and $C$ at first rise more steeply than A, and later turn and cross A. This is not so. As far as this very brief series of tests is concerned, light overconsolidation seems to be remembered by the slight tendency to higher stresses during the earlier stages of shearing as represented by the correction in equation (5), and by a greater tendency to dilate. This suggests that the correction in equation (5) may represent the breakdown by shearing of hardening wrought by the overconsolidation. This, in 
turn, suggests that equation (4) could be applied to a strain-softening material, $f$ being interpreted as the proportion of bonds yet to be broken.

During these tests there was some suspicion that a rapid reduction of cell pressure resulted in a softening of the material. This is to be expected, because the compressibility of water is greater than that of the solid skeleton of the soil. Thus, a rapid reduction of cell pressure would cause the water to expand more than the skeleton, forcing the particles apart, and breaking whatever bonds existed. Such an effect may occur around excavations which are dug quickly.

The analysis of normally consolidated slippery-penny-clays may be extended to volumetric effects by assuming that, on average, each turning event annihilates an equal piece of volume. Then

$$
\epsilon_{\mathrm{v}}=A^{*}\left(1-\mathrm{e}^{-a \epsilon}\right)
$$

in an obvious notation. As far as can be ascertained from these preliminary tests, equation (7) is reasonably accurate up to $80 \%$ failure stress.

These tests also suggest that, for the lightly overconsolidated condition, it may prove possible to represent the volumetric strain by

$$
\epsilon_{\mathrm{v}}=A^{*}\left(1-\mathrm{e}^{-a \epsilon}\right)-C^{*}\left(1-\mathrm{e}^{-c \epsilon}\right)
$$

where $A^{*}$ measures a contraction as in equation (7), and where $C^{*}$ measures dilation presumably associatcd with the appearance of micro-voids bctwcen and through domains (Smart, 1969).

Although large strains are involved here, all the strains discussed are, and are intended to be, the ratios of the changes of dimension to the original values of dimension.

The Writer thanks Messrs R. Laird and W. Thomson for much help with the tests.

\section{REFERENCES}

Bekker, M. G. (1955). Theory of land locomotion. University of Michigan Press.

Loudon, P. A. (1967). Some deformation characteristics of kaolin. $\mathrm{PhD}$ thesis, University of Cambridge.

Roscoe, K. H. \& Burland, J. B. (1968). On the generalized stress-strain behaviour of 'wet' clay. Engineering plasticity, 535-609. (Heyman, J. \& Leckie, F. A., eds.) Cambridge University Press.

Smart, P. (1966). Particle arrangements in kaolin. Clays Clay Miner. 15, 241-254.

Smart, P. (1967). Soil structure, mechanical properties and electron microscopy. $\mathrm{PhD}$ thesis, University of Cambridge.

Smart, P. (1969). Soil structure in the electron microscope. Structure, solid mechanics and engineering design, 249-255. (Te'eni, M., ed.) London: Wiley-Interscience.

Wroth, C. P. \& Loudon, P. A. (1967). The correlation of strains within a family of triaxial tests on overconsolidated samples of kaolin. Proc. Geotechn. Conf. Oslo 1967 on Shear Strength Properties of Natural Soils and Rocks, 159-163. Oslo: Norwegian Geotechnical Institute.

\section{Use of servo mechanisms for volume change measurement and $K_{0}$ consolidation}

LEWIN, P. I. (1971). Géotechnique 21, No. 3, 259-262. Discussion (1972) 22, No. 1, 186-187.

\section{R. D. Holtz, Swedish Geotechnical Institute, Stockholm}

The Author has presented an interesting improvement over the common method of observing volume changes by burettes during $K_{0}$ consolidation in the triaxial test. However, considerable personal experience with non-hydrostatic consolidation, e.g., Holtz and Krizek (1971), has shown that even though the average cross-sectional area of the specimen was calculated to 